and 0.69 per 1000 person-years, respectively. Patients with SLE had a higher risk of HP $(\mathrm{p}<0.001$, figure 1$)$. The ageand sex-adjusted hazard ratio (HR) was 2.04 (95\%CI;1.532.73). After adjusting for age, sex and baseline covariates, the HR was 1.85 (95\% CI;1.37-2.52). After adjusting for age, sex, baseline covariates, and weighted time-dependent covariates, the HR was 1.63 (95\% CI;1.07-2.50).

Conclusions Patients with a new diagnosis of SLE have 1.6 fold increased risk of HP than the general population. Given the impact of HP, this has important implications for mortality, functional status, and quality of life of people with SLE.

Funding Source(s): Canadian Institutes for Health Research (Grants MOP 125960 and THC 135235).

\section{SULFAMETHOXAZOLE AND TRIMETHOPRIM CAUSES TRUE LUPUS EXACERBATIONS RATHER THAN DRUG REACTION}

John T Berry, Rachel E Kneeland, Rami Martini, Sydney R Brandwein, Monika Starosta. Advocate Lutheran General Hospital

\subsection{6/lupus-2019-Ism.77}

Background Sulfamethoxazole and trimethoprim (TMP-SMX) is frequently used for urinary tract infections and Pneumocystis prophylaxis in patients on high dose systemic steroids or cyclophosphamide. Recommendations on avoiding TMP-SMX in systemic lupus erythematosus (SLE) are based on anecdotal evidence. Many authors describe adverse effects of TMP-SMX to be a drug reaction or allergy rather than a true SLE exacerbation.

Methods We performed chart review in an urban community clinic setting from 2013 to 2018.

Results Three patients were identified as having a lupus exacerbation within one week of exposure to TMP-SMX, and one patient within two months. Exacerbations consisted of fever and arthralgia, lupus enteritis, lupus enteritis with pericarditis, and inflammatory arthritis. Three cases occurred in the summer (two in June and one in September) and one case in the winter (December). All patients required hospitalization. Two of four patients had stable SLE prior to exacerbation. Symptoms in all patients resolved after treatment with high dose systemic glucocorticoids. There were no recurrent manifestations after TMP-SMX was stopped. All patients continued baseline medications and did not need additional long-term immunosuppression.

Conclusions TMP-SMX can cause severe exacerbations of SLE and should be avoided in these patients. To the best of our knowledge, this is the first report of two instances of TMP-

\begin{tabular}{llll} 
Abstract 77 Table 1 & & \\
\hline Demographics & Serology/APS & $\begin{array}{l}\text { Baseline } \\
\text { Medications }\end{array}$ & $\begin{array}{l}\text { Reaction to TMP- } \\
\text { SMX }\end{array}$ \\
\hline $47 \mathrm{~F}$ & Chromatin, Smith, RNP & Hydroxychloroquine & Fever, Arthralgia \\
$37 \mathrm{~F}$ & Chromatin, Smith, RNP & Belimumab & Lupus Enteritis \\
$56 \mathrm{M}$ & Chromatin, Cardiolipin IgM, & Hydroxychloroquine, & Inflammatory \\
& Beta 2 glycoprotein 1 IgM & Belimumab & Arthritis \\
$42 \mathrm{~F}$ & Chromatin, Smith, RNP, & Hydroxychloroquine, & Lupus Enteritis, \\
& dsDNA, Ribosomal P, SSA & Prednisone & Ascites, \\
& & & Pericarditis \\
\hline
\end{tabular}

Demographics, serologies, and manifestations of patients with lupus exacerbation
SMX induced lupus enteritis. Serologic associations may identify those with greater risk, as a positive RNP, Smith and chromatin antibodies were found in three patients and SSA was positive in only one patient. Increased photosensitivity secondary to TMP-SMX may lead to exacerbation, as three cases occurred during summer months. More studies are needed to clarify guidelines for TMP-SMX use in patients with SLE and promote awareness of exacerbation risk within the primary care community.

Funding Source(s): None

\section{DISCOVERY OF A NOVEL ANTI-TACI ANTIBODY WITH SLE TREATMENT EFFECT AND LIMITED SIDE-EFFECT}

Xiao Feng*, Dawei Sun, Suofu Qin, Tao Wang, Hongbao Yan, Guosheng Teng, Chong Che. Gene Science Pharmaceuticals

\subsection{6/lupus-2019-Ism.78}

Background All clinical trials involving the BAFF axis to date have targeted the ligand, BAFF. Remarkably, there have been no reports of targeting any of the BAFF receptors, BCMA, TACI, or BR3. TACI is critical for $\mathrm{T}$ cell-independent type antibody production and memory $B$ cell survival. Also IgG autoantibody production in SLE patient is mainly a $\mathrm{T}$ cellindependent. Thus, TACI is a promising target for SLE treatment.

Methods In this study, we discovered a novel anti-TACI antibody, GenSci-X002, by hybridoma and humanization approach to investigate its funtion on SLE treatment.

Results GenSci-X002 specifically binds to TACI receptor with sub-nanomolar affinity and blocks the binding of TACI and BAFF ligand. Also, GenSci-X002 neutralized BAFF-TACI downstream signal in a luciferase reporter assay. In SLE mouse model, GenSci-X002 exhibited equivalent SLE treatment effects with Belimumab, evidenced by the significant decrease of pathogenic autoantibody production, kidney Ig deposition and the development of nephritis. Remarkably, GenSci-X002 didnt alter normal $B$ cell subsets and B cell numbers with minimal side effect.

Conclusions Here, we show that anti-TACI antibody protected against BAFF-mediated autoimmune manifestations while preserving B cells, suggesting that loss of BAFF signaling through TACI rather than loss of $B$ cells may underpin the effect of Belimumab in the clinic. Therefore, B cell blockade of TACI by GenSciX002 may offer a more specific and safer therapeutic alternative to broad B cell depletion in SLE. The candidate therapeutics, GenSci-X002, will be further studied in preclinical evaluation to enter early-stage clinical trials of SLE in future.

Funding Source(s): None

\section{CLINICAL CHARACTERISTICS OF PATIENTS WITH SYSTEMIC LUPUS ERYTHEMATOSUS COMPLICATED WITH PULMONARY THROMBOEMBOLISM}

Hong Zhu*, Yu-Jia Jing, Yan Zhou, Yao Chen. Department of Rheumatology, General Hospital of Ningxia Medical University. Yinchuan 750004, China

\subsection{6/lupus-2019-Ism.79}

Background There is strong evidence for an association between SLE and an increased risk of pulmonary thromboembolism (PTE).PTE occurs with a higher frequency in SLE patients compared to the general population. 